\title{
Symbolic Parametric Safety Analysis of Linear Hybrid Systems with BDD-Like Data-Structures ${ }^{\star}$
}

\author{
Farn Wang \\ Dept. of Electrical Engineering, National Taiwan University \\ 1, Sec. 4, Roosevelt Rd., Taipei, Taiwan 106, ROC \\ Phone: +886-2-23635251 ext. 435; Fax: +886-2-23671909 \\ farn@cc.ee.ntu.edu.tw \\ http://cc.ee.ntu.edu.tw/ farn
}

Model-checker/simulator red 5.3 is available at http://cc.ee.ntu.edu.tw/ ${ }^{\sim}$ val

\begin{abstract}
We introduce a new BDD-like data structure called HybridRestriction Diagrams (HRDs), for the representation and manipulation of linear hybrid automata (LHA) state-spaces, and present algorithms for weakest precondition calculations. This permits us to reason about the valuations of parameters that make safety properties satisfied. Advantages of our approach include the ability to represent discrete state information and concave polyhedra in a unified scheme as well as to save both memory consumptions and manipulation times, when processing the same substructures in state-space representations. Our experimental results document its efficiency in practice.
\end{abstract}

Keywords: data-structures, BDD, hybrid automata, verification, modelchecking

\section{Introduction}

Linear hybrid automata (LHA) are state-transition systems equipped with continuous variables that can change values with different rates [6]. They are important to computing and society because of their extensive modeling capability. In practice, such models are usually presented with symbolic constants, called $p a-$ rameters, whose values may engender different behaviors of the models. Setting and calibrating these parameters is a crucial task for the engineers developing hybrid systems. Parametric safety analysis (PSA) of LHA can generate a symbolic characterization of the parameters' valuations, called solutions, that make a model satisfy a safety property. Such a symbolic characterization, once constructed, sheds important feedback information to engineers and can be used

\footnotetext{
* The work is partially supported by NSC, Taiwan, ROC under grants NSC 92-2213E-002-103 and NSC 92-2213-E-002-104. We would like to thank the TReX team, especially Mihaela Sighireanu and Aurore Collomb-Annichini, for kindly implementing a TReX version without the reduce package for us.
} 
repeatedly in the synthesis and verification of implementations of the corresponding model.

Although, the emptiness problem of PSA solution spaces is undecidable in general [7], people have constructed experimental tools for the verification of LHA $[1,3,4,6]$ based on the following three reasons. First, the verification problem is by itself interesting and challenging. Second, it may happen that there exist techniques and strategies which can solve many typical examples. Third, there is still an urgent need in industry for such tools to help engineers with fast prototyping of their compelx designs.

Because LHA necessarily involves continuous variables, it is helpful and more efficient to represent and manipulate their state-space symbolically. Others have developed separate representation schemes for the discrete part and continuous part of LHA $[1,3,4,6]$. According to previous experiences $[9,11,17-19]$, it can be more efficient to have a unified data-structure for both the discrete part and the continuous part. Moreover, the schemes used in $[1,3,4,6]$ only represent convex polyhedra in LHA state-space ( concave polyhedra have to be represented as sets of convex ones) and suffer from the limitation to share common representation structures in different convex polyhedra.

In this work, we extend BDD [9,11] for the representation and manipulation of LHA state-spaces. A BDD [11] is an acyclic graph, with a single source and two sinks for true and false respectively. Each internal node is labeled with a Boolean decision atom and has a true-child and a false-child. A BDD works as a decision diagram for state-space membership. Any sequence of decision atoms labeled on a path in a BDD must follow a predefined total ordering.

The term BDD-like data-structure is coined in [19] to call the many BDD extensions in the literature. BDD-like data-structures have the advantage of datasharing in both representation and manipulation and have shown great success in VLSI verification industry. The same structure following two decision path prefixes will only be represented once. Also, a pattern of operand substructures will only be manipulated once. The manipulation result will be saved and returned immediately the next time when the same pattern is encountered. These features of BDD-technology lead to not only savings in memory consumptions but also speed-up in manipulations.

One of the major difficulties to use BDD-like data-structures to analyze LHAs comes from the unboundedness of the dense variable value ranges and the unboundedness of linear constraints. To explain one of the major contribution of this work, we need to discuss the following issue first. In the research of BDD-like data-structures, there are two classes of variables: system variables and decision atoms [19]. System variables are those used in the input behavior descriptions. Decision atoms are those labeled on each BDD nodes. For discrete systems, these two classes are the same. But for dense-time systems, decision atoms can be different from state variables. For example, in CDD (Clock-Difference Diagram) [10] and CRD (Clock-Restriction Diagram) [19], decision atoms are of the form $x-x^{\prime}$ where $x$ and $x^{\prime}$ are system variables of type clock. Previous work on BDD-like data-structures are based on the assumption that decision atom domains are of finite sizes. Thus we need new techniques to extend BDD-like data-structures 
to represent and manipulate state-spaces of LHAs. Our innovations include using constraints, like $-3 A+x-4 y$ (where $A, x, y$ are dense system variables), as the decision atoms and using total dense orderings among these atoms. In this way, we devise HRD (Hybrid-Restriction Diagram) and successfully extend BDD-technology to models with unbounded domains of decision atoms.

In total, we defined three total dense orderings for HRD decision atoms (section 5). We also present algorithms for set-oriented operations (section 6) and symbolic weakest precondition calculation (section 7), and a procedure for symbolic parametric safety analysis (section 7 ). We have also developed a technique, which prunes state-space exploration based on parameter space characterization and enhances the performance of our tool by orders of magnitude (section 8). Desirably, this technique does not sacrifice the precision of parametric safety analysis. Especially, for one benchmark in our experiments (see section 9), the state-space exploration does not converge without this technique! To our knowledge, nobody else has come up with a similar technique. Finally, we have implemented our ideas in our tool red 5.0 and reported our experiments to see how the three dense-orderings perform and how our implementation performs in comparison with HyTech 2.4.5 [13] and TReX 1.3 [1,3].

\section{Related Work}

People have used convex subspaces, called convex polyhedra, as basic unit for symbolic manipulation. A convex polyhedron characterizes a state-space of an LHA and can be symbolically represented by a set of constraints like $a_{1} x_{1}+$ $\ldots+a_{n} x_{n} \sim c[4-6]$. Two commonly used representations for convex polyhedra in HyTech are polyhedras and frames in dense state-space [13]. These two representations neither are BDD-like nor can represent concave state-spaces. Data-sharing among convex polyhedra is difficult.

In 1993 [22], Wang, Mok, and Emerson discussed how to use BDD with decision atoms like $x_{i}+c \leq x_{j}+d$ to model-check timed automata. In the last several years, people have explored in this approach in the hope of duplicating the success of BDD techniques $[9,11]$ in hardware verification for the verification of timed automata $[2,8,10,14-19]$. Especially, our HRD can be seen as variation of CDD [10] and extension of CRD [17,18] for timed systems. In [10], CDD only served as a recording device in that reachable state-space representations were analyzed using DBM [12] and then converted to CDD. In [19-21], a full set of verification algorithms (including forward/backward reachability analysis, normalization, and full TCTL model-checking procedures) for CRD were reported.

For parametric safety analysis, Annichini et al have extended DBM [12] to PDBM for parametric safety analysis of timed automata [1,3] and implemented a tool called $T R e X$, which also supports verification with lossy channels.

\section{Parametric Safety Analysis of Linear Hybrid Automata}

A linear hybrid automaton (LHA) [6] is a finite-state automaton equipped with a finite set of dense system variables which can hold real-values. At any moment, 


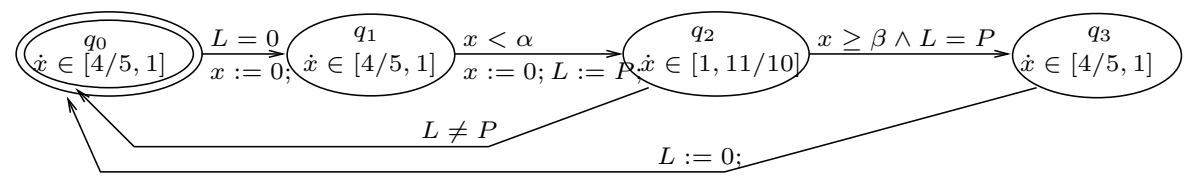

Fig. 1. Fischer's timed mutual exclusion algorithm in LHA

the LHA can stay in only one mode (or control location). In its operation, one of the transitions can be triggered when the corresponding triggering condition is satisfied. Upon being triggered, the LHA instantaneously transits from one mode to another and sets some dense variables to values in certain ranges. In between transitions, all dense variables increase their readings at rates determined by the current mode. Since LHA has been well-studied in the literature, we leave its definition in our full version report. In figure 1, we have drawn a version of the Fischer's mutual exclusion algorithm for a process. There are two parameters $\alpha$ and $\beta$ that control the behavior of the processes. In each mode, local clock $x$ increases its reading according to a rate in $[4 / 5,1]$ or $[1,11 / 10]$.

Our verification framework is called parametric safety analysis (PSA) problem. Such a problem instance, denoted $\operatorname{PSA}(A, \eta)$, consists of an LHA $A$ and a safety state-predicate $\eta$ and asks for a symbolic characterization of all parameter valuations, called solutions, that make all reachable states satisfy $\eta$. The general parametric safety analysis problem can be proved incomputable with a straightforward adaptation of the undecidability proof in [7].

\section{HRD (Hybrid-Restriction Diagram)}

For efficiency of manipulation, we have the following requirements on constraints used to represent a convex polyhedron. Given a constraint like $\sum_{i} a_{i} x_{i} \sim c,(1)$ $a_{i}$ 's are integers such that $\operatorname{gcd}\left\{a_{i} \mid 1 \leq i \leq n ; a_{i} \neq 0\right\}=1,(2) x_{1}, \ldots, x_{n}$ are system variables, $(3) \sim \in\{$ " $\leq ", "<"\}$, and (4) $c$ is either a rational number or $\infty$ such that when $c=\infty, \sim=$ " $<$ ". We shall call " $\sum_{i} a_{i} x_{i}$ " an LH-expression and "( $\sim, c)$ " an LH-upperbound. Formally, a convex polyhedron can be defined as a mapping from the set of LH-expressions to the set of LH-upperbounds. For a given $X$, the set of all LH-expressions and the set of convex polyhedra are both infinite. For any two $(\sim, c)$ and $\left(\sim^{\prime}, c^{\prime}\right),(\sim, c)$ is more restrictive than $\left(\sim^{\prime}, c^{\prime}\right)$, denoted $(\sim, c) \sqsubset\left(\sim^{\prime}, c^{\prime}\right)$, iff $c<c^{\prime}$ or $\left(c=c^{\prime} \wedge \sim="<" \wedge \sim^{\prime}=" \leq "\right)$.

To construct BDD-like data-structures, three fundamental issues have to be solved. The first is the domain of the decision atoms; the second is the range of the arc labels from BDD nodes; and the third is the evaluation ordering among the decision atoms. For modularity of presentation, we shall leave the discussion of the evaluation orderings to section 5. In this section, we shall assume that we are given a decision atom evaluation ordering.

We decide to follow an approach similar to the one adopted in [19]. That is, the decision atoms of HRD are LH-expressions and the arcs are labeled with LH-upperbounds. A node label $\sum_{i} a_{i} x_{i}$ with a corresponding outgoing arc label 


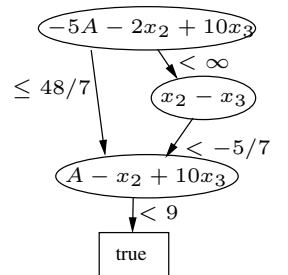

(a) HRD with coefficient ordering

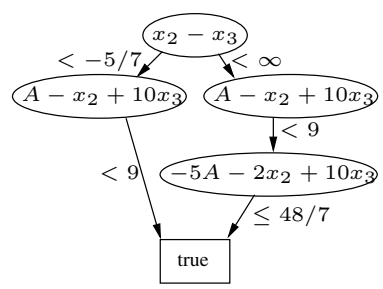

(b) HRD with magnitude ordering

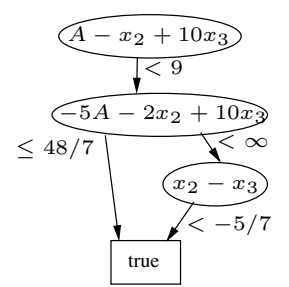

(c) HRD with dictionary ordering

Fig. 2. Examples of HRD

$(\sim, c)$ constitute the constraint of $\sum_{i} a_{i} x_{i} \sim c$. A source-to-sink path in an HRD thus represents the conjunction of constituent constraints along the path. Figure 2(a) is an HRD example for the concave space of

$$
\left(x_{2}-x_{3} \leq-5 / 7 \vee-5 A-2 x_{2}+10 x_{3} \leq 48 / 7\right) \wedge A-x_{2}+10 x_{3}<9
$$

assuming that $-5 A-2 x_{2}+10 x_{3}$ precedes $x_{2}-x_{3}$ (in symbols $-5 A-2 x_{2}+10 x_{3} \prec$ $x_{2}-x_{3}$ ) and $x_{2}-x_{3}$ precedes $A-x_{2}+10 x_{3}$ in the given evaluation ordering. In this example, the system variables are $A, x_{2}, x_{3}$ while the decision atoms are $x_{2}-x_{3},-5 A-2 x_{2}+10 x_{3}$, and $A-x_{2}+10 x_{3}$.

Definition 1. HRD (Hybrid-Restriction Diagram) Given a dense variable set $X=\left\{x_{1}, \ldots, x_{n}\right\}$ and an evaluation ordering $\prec$ among normalized LH-expressions of $X$, an $H R D$ is either true or a tuple $\left(v,\left(\beta_{1}, D_{1}\right), \ldots,\left(\beta_{m}, D_{m}\right)\right)$ such that

- $v$ is a normalized LH-expression;

- for each $1 \leq i \leq m, \beta_{i}$ is an LH-upperbound s.t. $(<, \infty) \neq \beta_{1} \sqsubset \beta_{2} \sqsubset \ldots \sqsubset$ $\beta_{m} ;$ and

- for each $1 \leq i \leq m, D_{i}$ is an HRD such that if $D_{i}=\left(v_{i}, \ldots\right)$, then $v \prec v_{i}$. For completeness, we use "()" to represent the HRD for false.

In our algorithms, false does not participate in comparison of evaluation orderings among decision atoms. Also, note that in figure 2, for each arc label $(\sim, c)$, we simply put down $\sim c$ for convenience.

\section{Three Dense Orderings among Decision Atoms}

In the definition of a dense-ordering among decision atoms (i.e., LH-expressions), special care must be taken to facilitate efficient manipulation of HRDs. Here we use the experience reported in [19] and present three heuristics in designing the orderings among LH-expressions. The three heuristics are presented in sequence proportional to their respective importances.

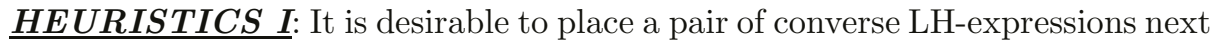
to one another so that simple inconsistencies can be easily detected. That is, LHexpressions $\sum_{i} a_{i} x_{i}$ and $\sum_{i}-a_{i} x_{i}$ are better placed next to one another in the ordering. With this arrangement, inconsistencies like $-x_{1}+3 x_{2} \leq-5 \wedge x_{1}-3 x_{2}<$ 0 can be checked by comparing adjacent nodes in HRD paths. To fulfill this 
requirement, when comparing the precedence between LH-expressions in a given ordering, we shall first toggle the signs of coefficients of an LH-expression if its first nonzero coefficient is positive. If two LH-expressions are identical after the necessary toggling, then we compare the signs of their first nonzero coefficients to decide the precedence between the two.

With this heuristics, from now on, we shall only focus on the orderings among LH-expressions whose first nonzero coefficients are negative.

$\underline{\boldsymbol{H E U R I S T I C S ~ I I}}$ : According to the literature, it is important to place strongly correlated LH-expressions close together in the evaluation orderings. Usually, instead of a single global LHA, we are given a set of communicating LHAs, each representing a process. Thus it is desirable to place LH-expressions for the same process close to each other in the orderings. Our second heuristics respects this experience. Given a system with $m$ processes with respective local dense variables, we shall partition the LH-expressions into $m+1$ groups: $G_{0}, G_{1}, \ldots, G_{m}$. $G_{0}$ contains all LH-expressions without local variables (i.e., coefficients for local variables are all zero). For each $p>0, G_{p}$ contains all LH-expressions with a nonzero coefficient for a local variable of process $p$ and only zero coefficients for local variables of processes $p+1, \ldots, m$. Then our second heuristics requires that for all $0 \leq p<m$, LH-expressions in $G_{p}$ precede those in $G_{p+1}, \ldots, G_{m}$.

$\underline{\boldsymbol{H E U R I S T I C S ~ I I I}}$ : If the precedence between two LH-expressions cannot be determined with heuristics I and II, then the following third heuristics comes to play. The design of this heuristics is a challenge since each of $G_{0}, \ldots, G_{m}$ can be of infinite size. Traditionally, BDD-like data-structures have been used with finite decision atom domains. Carefully examining the BDD manipulation procedures, we found that the sizes of the domains do not matter. What really matters is the ordering among all decision atoms so that we know which atoms is to be decided next along a decision path. Thus, we invent to use the following three dense-orderings among LH-expressions.

Dictionary Ordering: We can represent each LH-expression as a string, assuming that the ordering among $x_{1}, \ldots, x_{n}$ is fixed and no blanks are used in the string. Then we can use dictionary ordering and ASCII ordering to decide the precedence among LH-expressions. For the LH-expressions in figure 2, we then have $-5 A-2 x_{2}+10 x_{3} \prec A-x_{2}+10 x_{3} \prec x_{2}-x_{3}$ since '-' precedes ' $A$ ' and ' $A$ ' precedes ' $x$ ' in ASCII. The corresponding HRD in dictionary ordering is in figure 2(c). One interesting feature of this ordering is that it has the potential to be extended to nonlinear hybrid constraints. For example, we may say $\cos \left(x_{1}\right)+x_{2}^{3} \prec x_{2}^{2}-x_{2} x_{3}$ in dictionary ordering since ' $c$ ' precedes ' $x$ ' in ASCII.

Coefficient Ordering: Assume that the ordering of the dense variables is fixed as $x_{1}, \ldots, x_{m}$. In this ordering, the precedence between two LH-expressions is determined by iteratively comparing the coefficients of dense variables $x_{1}, \ldots, x_{n}$ in sequence. For the LH-expressions in figure 2, we then have $-5 A-2 x_{2}+10 x_{3} \prec$ $x_{2}-x_{3} \prec A-x_{2}+10 x_{3}$. The HRD in this ordering is in figure $2(\mathrm{a})$.

Magnitude Ordering: This ordering is similar to the last one. Instead of comparing coefficients, we compare the absolute values of coefficients. We iteratively 
- first compare the absolute values of coefficients of $x_{i}$, and

- if they are equal, then compare the signs of coefficients of $x_{i}$.

For the LH-expressions in figure $2, x_{2}-x_{3} \prec A-x_{2}+10 x_{3} \prec-5 A-2 x_{2}+10 x_{3}$ in this magnitude ordering. The corresponding HRD is in figure 2(b).

\section{Set-Oriented Operations}

Please be reminded that an HRD records a set of convex polyhedra. For convenience of discussion, given an HRD, we may just represent it as the set of convex polyhedra recorded in it. Definitions of set-union $(\cup)$, set-intersection $(\cap)$, and set-exclusion $(-)$ of two convex polyhedra sets respectively represented by two HRDs are straightforward. For example, given HRDs $D_{1}:\left\{\zeta_{1}, \zeta_{2}\right\}$ and $D_{2}:\left\{\zeta_{2}, \zeta_{3}\right\}, D_{1} \cap D_{2}$ is the HRD for $\left\{\zeta_{2}\right\} ; D_{1} \cup D_{2}$ is for $\left\{\zeta_{1}, \zeta_{2}, \zeta_{3}\right\}$; and $D_{1}-D_{2}$ is for $\left\{\zeta_{1}\right\}$. The complexities of the three manipulations are all $O\left(\left|D_{1}\right| \cdot\left|D_{2}\right|\right)$.

Given two convex polyhedra $\zeta_{1}$ and $\zeta_{2}, \zeta_{1} \wedge \zeta_{2}$ is a new convex polyhedron representing the space-intersection of $\zeta_{1}$ and $\zeta_{2}$. Formally speaking, for decision atom $\sum_{i} a_{i} x_{i}, \zeta_{1} \wedge \zeta_{2}\left(\sum_{i} a_{i} x_{i}\right)=\zeta_{1}\left(\sum_{i} a_{i} x_{i}\right)$ if $\zeta_{1}\left(\sum_{i} a_{i} x_{i}\right) \sqsubset \zeta_{2}\left(\sum_{i} a_{i} x_{i}\right)$; or $\zeta_{2}\left(\sum_{i} a_{i} x_{i}\right)$ otherwise. Space-intersection $(\wedge)$ of two HRDs $D_{1}$ and $D_{2}$, in symbols $D_{1} \wedge D_{2}$, is a new HRD for $\left\{\zeta_{1} \wedge \zeta_{2} \mid \zeta_{1} \in D_{1} ; \zeta_{2} \in D_{2}\right\}$. Our current algorithm of the manipulation has complexity $O\left(\left|D_{1}\right|^{2}\left|D_{2}\right|^{2}\right)$.

Given an evaluation ordering, we can write HRD-manipulation algorithms pretty much as usual $[9,11,15,19]$. For convenience of presentation, we may represent an $\operatorname{HRD}\left(u,\left(\beta_{1}, B_{1}\right), \ldots,\left(\beta_{n}, B_{n}\right)\right)$ symbolically as $\left(u,\left(\beta_{i}, B_{i}\right)_{1 \leq i \leq n}\right)$. A union operation union $(B, D)$ can then be implemented as follows.

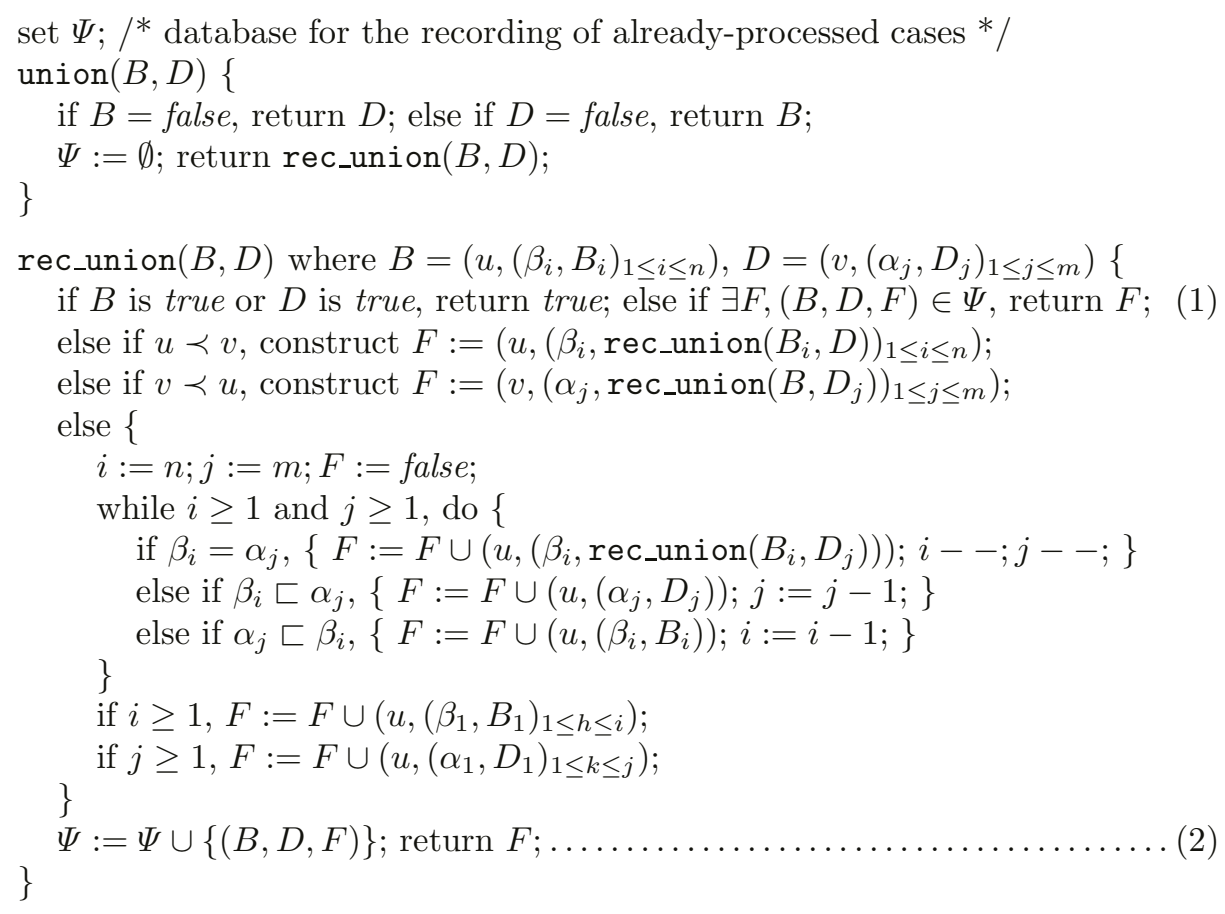


Note in statement (1), we take advantage of the data-sharing capability of HRDs so that we do not process the same substructure twice. Set $\Psi$ is maintained in statement (2) to record the processing result the first time the substructure is encountered. The algorithms for $\cap$ and - are pretty much the same. The one for space intersection $\wedge$ is much more involved and is not discussed here.

\section{Weakest Preconditon Calculation and Symbolic PSA}

Our tool red 5.0 runs a backward reachability analysis by default. Suppose we are given an LHA $A$. There are two basic procedures in this analysis procedure. The first, xtion $(D, e)$, computes the weakest precondition from state-space represented by HRD $D$ through discrete transition $e$ from mode $q$ to $q^{\prime}$. Assume that the variables that get assigned in $e$ are $y_{1}, \ldots, y_{k}$ and there is no variable that gets assigned twice in $e$. The characterization of $\operatorname{xtion}(D, e)$ is

$$
\mu(q) \wedge \tau(e) \wedge \exists y_{1} \ldots \exists y_{k}\left(D \wedge \wedge_{1 \leq i \leq k} y_{i} \in \pi\left(e, y_{i}\right)\right)^{1}
$$

Assume that delta_exp $(D)$ is the same as $D$ except that all dense variables $x$ are replaced by $x+\delta_{x}$ respectively. Here $\delta_{x}$ represents the value-change of variable $x$ in time-passage. For example, delta_exp $\left(2 x_{1}-3 x_{2} \leq 3 / 5\right)=2 x_{1}+$ $2 \delta_{x_{1}}-3 x_{2}-3 \delta_{x_{2}} \leq 3 / 5$.

Assume that for each dense variable $x, \gamma(q, x)$ specifies the rate interval of $x$ in $q$. The second basic procedure, time $(D, q)$, computes the weakest precondition from $D$ through time passage in mode $q$. It is characterized as

$$
\mu(q) \wedge \exists \delta_{x_{1}} \exists \delta_{x_{2}} \ldots \exists \delta_{x_{n}} \exists \delta\left(\begin{array}{c}
\delta \geq 0 \wedge \text { delta_exp }(D) \\
\wedge \wedge_{1 \leq i \leq n ; \gamma\left(q, x_{i}\right)=\left\langle d_{i}, d_{i}^{\prime}\right\rangle} \delta_{x_{i}} \in\left\langle d_{i} \delta, d_{i}^{\prime} \delta\right\rangle
\end{array}\right)
$$

One basic building block of both xtion() and time() is for the evaluation of $\exists x(D(x))$ and is implemented as follows.

$$
\exists x(D(x)) \equiv \text { var_del(xtivity }(D, x),\{x\}) .
$$

Procedure var_del $\left(D, X^{\prime}\right)$ eliminates all constraints in $D$ involving variables in set $X^{\prime}$. Procedure xtivity $(D, x)$ adds to a path every constraint that can be transitively deduced from two peer constraints involving $x$ in the same path in $D$. The algorithm of $x \operatorname{tivity}()$ is as follows.

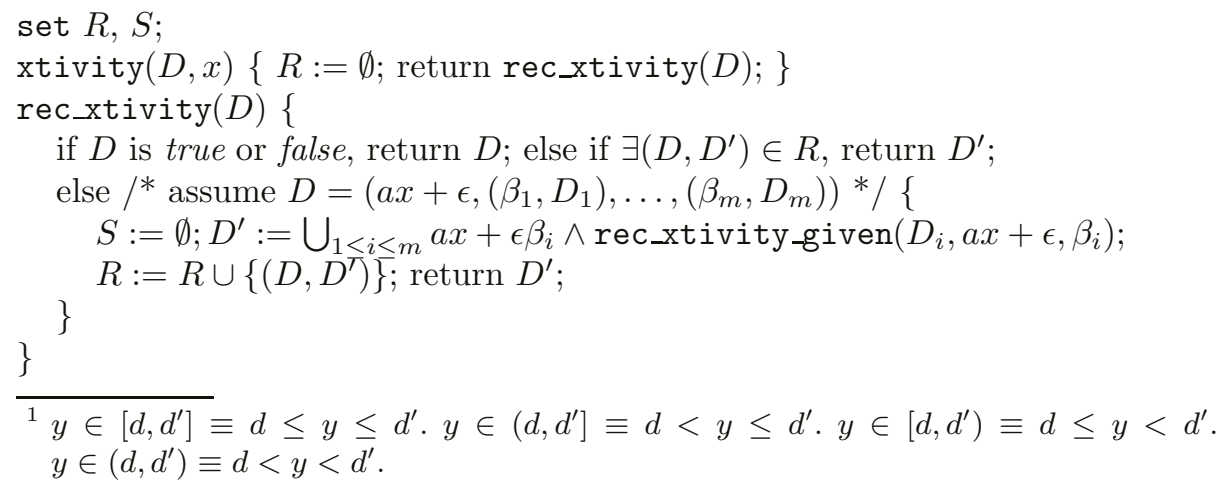


rec_xtivity_given $(D, a x+\epsilon, \beta)\{$

if $D$ is true or false, return $D$; else if $\exists\left(D, D^{\prime}\right) \in S$, return $D^{\prime}$; else ${ }^{*}$ assume $D=\left(b x+\epsilon^{\prime},\left(\beta_{1}, D_{1}\right), \ldots,\left(\beta_{m}, D_{m}\right)\right) * /\{$

if $a b<0$,

$$
D^{\prime}:=\bigcup_{1 \leq i \leq m}\left(\begin{array}{c}
b x+\epsilon^{\prime} \beta_{i} \wedge \text { rec_xtivity_given }\left(D_{i}, a x+\epsilon, \beta\right) \\
\wedge|b| \epsilon / \operatorname{gcd}(a, b)+|a| \epsilon^{\prime} / \operatorname{gcd}(a, b)\left(\left(|b| \beta+|a| \beta_{i}\right) / \operatorname{gcd}(a, b)\right)
\end{array}\right) ;
$$

else $D^{\prime}:=\bigcup_{1 \leq i \leq m} b x+\epsilon^{\prime} \beta_{i} \wedge$ rec_xtivity_given $\left(D_{i}, a x+\epsilon, \beta\right)$;

$S:=S \cup\left\{\left(D, D^{\prime}\right)\right\}$; return $D^{\prime}$;

\}

\}$/^{*}\left(|b| \beta+|a| \beta_{i}\right) / \operatorname{gcd}(a, b)$ is a shorthand for the new upperbound obtained from the xtivity of $a x+\epsilon \beta$ and $b x+\epsilon^{\prime} \beta_{i}{ }^{*} /$

Thus we preserve all constraints transitively deducible from a dense variable before it is eliminated from a predicate. This guarantees that no information will be unintentionally lost after the variable elimination.

Note that in our algorithm, we do not enumerate all paths in HRD to carry out this least fixpoint evaluation. Instead, in statement (3), our algorithm follows the traditional BDD programming style which takes advantage of the datasharing capability of BDD-like data-structures. Thus our algorithm does not explode due to the combinatorial complexity of path counts in HRD. This can be justified by the performance of our implementation reported in section 9 .

Assume that the transition set is $E$ and the unsafe states are in mode $q_{f}$. With the two basic procedures, then the backward reachable state-space from the unsafe states in $\neg \eta$ (represented as an HRD) can be characterized by

$$
\operatorname{lfp} Z .\left(\operatorname{time}\left(\neg \eta, q_{f}\right) \cup \bigcup_{e=\left(q, q^{\prime}\right) \in E} \operatorname{time}(\operatorname{xtion}(Z, e), q)\right)
$$

Here $\operatorname{lfp} Z . F(Z)$ is the least fixpoint of function $F()$ and is very commonly used in the reachable state-space representation of discrete and dense-time systems. After the fixpoint is successfully constructed, we conjunct it with the initial condition and then eliminate all variables except those parameters (geometrically speaking, projecting the reachable state-space to the dimensions of the parameters). Suppose the set of dense parameters is $H$. The characterization of unsafe parameter valuations is thus

$$
\operatorname{var} \_d e l\left(I \wedge \operatorname{lfp} Z .\left(\operatorname{time}\left(\neg \eta, q_{f}\right) \cup \bigcup_{e=\left(q, q^{\prime}\right) \in E} \operatorname{time}(\operatorname{xtion}(Z, e), q)\right), X-H\right)
$$

The solution space is characterized by the complement of this final result.

In our experience, normalizing convex polyhedron representations in HRDs may consume most of the resources. At this moment, our implementation does not enforce a normal form. We only try to eliminate some redundancies that can be derived from two peer constraints in the same convex polyhedron.

Polyhedron containment checking in an HRD is also important in controlling the size of the HRD. Specifically, we want to check if a path in the HRD specifies a polyhedra that is subsumed by another path's HRD. In [19], a sufficient condition called SCR (Straightforward Containment Requirement) was presented for the containment checking of zones. We have adapted SCR for HRDs. Specifically, given $D=\left(v,\left(\alpha_{j}, D_{j}\right)_{1 \leq j \leq m}\right), \operatorname{SCR}(D)=\left(v,\left(\alpha_{j}, D_{j}-\bigcup_{j<k \leq m} D_{k}\right)_{1 \leq j \leq m}\right)$. 
Lemma 1. Given any $H R D D, D$ and $\operatorname{SCR}(D)$ characterize the same real space.

In our current implementation, when we need to eliminate polyhedras contained by peer polyhedras in an HRD, we perform SCR to each node in the HRD in a bottom-up fashion.

\section{Pruning Strategy Based on Parameter Space Construction (PSPSC)}

We have also experimented with techniques to improve the efficiency of parametric safety analysis. One such technique, called $P S P S C$, is avoiding new statespace exploration if the exploration does not contribute to new parametric solutions. A constraint is static iff all its dense variables are parameters. Static constraints do not change their truth values. Once a static constraint is derived in a convex polyhedron, its truth value will be honored in all weakest preconditions derived from this convex polyhedron. All states backwardly reachable from a convex polyhedron must also satisfy the static constraints required in the polyhedron. Thus if we know that static parameter valuation $\mathcal{H}$ is already in the parametric solution space, then we really do not need to explore those states whose parameter valuations fall in $H$.

With PSPSC, our new parametric safety analysis procedure is as follows.

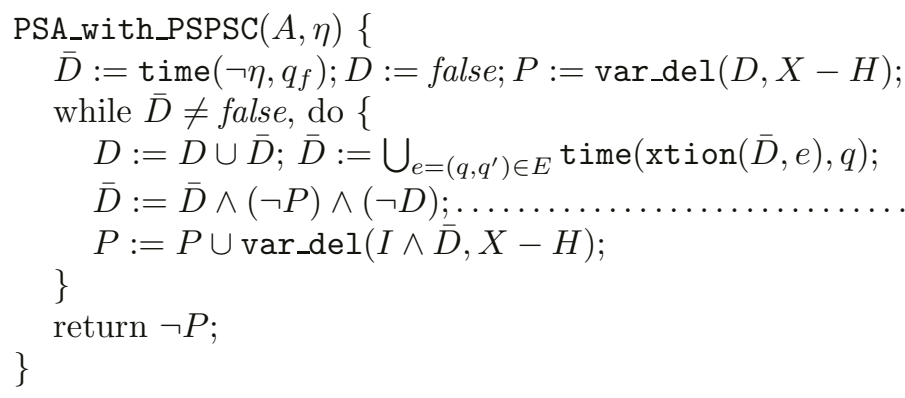

In the procedure, we use variable $P$ to symbolically accumulate the parametric evaluations leading to the unsafe states in the least fixpoint iterations. In statement (5), we check and eliminate in $\bar{D}$ those state descriptions which cannot possibly contribute to new parametric evaluations by conjuncting $\bar{D}$ with $\neg P$.

One nice feature of PSPSC is that it does not sacrifice the precision of our parametric safety analysis.

Lemma 2. $\mathcal{H}$ is a parametric solution to $A$ and $\eta$ iff $\mathcal{H}$ satisfies the return result of PSA_with_PSPSC $(A, \eta)$.

Proof: Note that the intersection at line (5) in procedure PSA_with_PSPSC() only stops the further exploration of those states that do not contribute to new parameter-spaces. Those parameter-spaces pruned in line (5) do not contribute 
Table 1. Comparison with HyTech w.r.t. number of processes

\begin{tabular}{|c|c|c|c|c|c|c|c|c|}
\hline \multirow[t]{3}{*}{ direction } & \multirow[t]{3}{*}{ benchmarks } & \multirow{3}{*}{$\begin{array}{l}\text { rates } \\
\text { used }\end{array}$} & \multirow{3}{*}{$m$} & \multirow{3}{*}{$\begin{array}{c}\text { HyTech } \\
2.4 .5\end{array}$} & \multicolumn{4}{|c|}{ red 5.0 (backward) } \\
\hline & & & & & dictionary & coefficient & magnitude & \multirow{2}{*}{$\begin{array}{c}\text { coefficient } \\
\text { PSPSC } \\
\end{array}$} \\
\hline & & & & & \multicolumn{3}{|c|}{ no PSPSC } & \\
\hline \multirow[t]{19}{*}{ backward } & \multirow{5}{*}{$\begin{array}{l}\text { Fischer's } \\
\text { mutual } \\
\text { exclusion } \\
\text { ( } m \\
\text { processes }) \\
\end{array}$} & \multirow{5}{*}{$\begin{array}{c}{[4 / 5,1]} \\
{[1,11 / 10]}\end{array}$} & 2 & $0.23 \mathrm{~s}$ & $0.10 \mathrm{~s} / 17 \mathrm{k}$ & $0.11 \mathrm{~s} / 17 \mathrm{k}$ & $0.11 \mathrm{~s} / 17 \mathrm{k}$ & $0.07 \mathrm{~s} / 16 \mathrm{k}$ \\
\hline & & & 3 & $2.40 \mathrm{~s}$ & $1.83 \mathrm{~s} / 81 \mathrm{k}$ & $1.75 \mathrm{~s} / 74 \mathrm{k}$ & $1.23 \mathrm{~s} / 59 \mathrm{k}$ & $0.70 \mathrm{~s} / 44 \mathrm{k}$ \\
\hline & & & 4 & $28.04 \mathrm{~s}$ & $20.29 \mathrm{~s} / 320 \mathrm{k}$ & $23.85 \mathrm{~s} / 269 \mathrm{k}$ & $12.38 \mathrm{~s} / 215 \mathrm{k}$ & $5.14 \mathrm{~s} / 163 \mathrm{k}$ \\
\hline & & & 5 & $\mathrm{O} / \mathrm{M}$ & $278.8 \mathrm{~s} / 1420 \mathrm{k}$ & $354.1 \mathrm{~s} / 1149 \mathrm{k}$ & $162.0 \mathrm{~s} / 1034 \mathrm{k}$ & $31.36 \mathrm{~s} / 474 \mathrm{k}$ \\
\hline & & & 6 & $\mathrm{O} / \mathrm{M}$ & $2846 \mathrm{~s} / 5848 \mathrm{k}$ & $9923 \mathrm{~s} / 8796 \mathrm{k}$ & $1485 \mathrm{~s} / 4000 \mathrm{k}$ & $168.6 \mathrm{~s} / 1170 \mathrm{k}$ \\
\hline & GRC & \begin{tabular}{|l|l|}
0,0 & $, 1,1$ \\
\end{tabular} & 2 & $\mathrm{O} / \mathrm{M}$ & $0.79 \mathrm{~s} / 103 \mathrm{k}$ & $0.68 \mathrm{~s} / 101 \mathrm{k}$ & $0.68 \mathrm{~s} / 101 \mathrm{k}$ & $0.76 \mathrm{~s} / 94 \mathrm{k}$ \\
\hline & (gate & {$[9,10]$} & 3 & $\mathrm{O} / \mathrm{M}$ & $11.48 \mathrm{~s} / 806 \mathrm{k}$ & $8.85 \mathrm{~s} / 616 \mathrm{k}$ & $8.84 \mathrm{~s} / 616 \mathrm{k}$ & $11.48 \mathrm{~s} / 530 \mathrm{k}$ \\
\hline & + controller & {$[-10,-9]$} & 4 & $\mathrm{O} / \mathrm{M}$ & $248.5 \mathrm{~s} / 6046 \mathrm{k}$ & $184.9 \mathrm{~s} / 4249 \mathrm{k}$ & $186.1 \mathrm{~s} / 4249 \mathrm{k}$ & $252.5 \mathrm{~s} / 2820 \mathrm{k}$ \\
\hline & $+m$ trains $)$ & & 5 & O/M & $6095 \mathrm{~s} / 37093 \mathrm{k}$ & $4883 \mathrm{~s} / 25841 \mathrm{k}$ & $4900 \mathrm{~s} / 25841 \mathrm{k}$ & $6527 \mathrm{~s} / 19234 \mathrm{k}$ \\
\hline & reactor & {$[1,1]$} & 2 & $0.056 \mathrm{~s}$ & $0.08 \mathrm{~s} / 19 \mathrm{k}$ & $0.07 \mathrm{~s} / 19 \mathrm{k}$ & $0.06 \mathrm{~s} / 19 \mathrm{k}$ & $0.05 \mathrm{~s} / 15 \mathrm{k}$ \\
\hline & (controller & & 3 & $0.33 \mathrm{~s}$ & $0.41 \mathrm{~s} / 51 \mathrm{k}$ & $0.38 \mathrm{~s} / 52 \mathrm{k}$ & $0.37 \mathrm{~s} / 52 \mathrm{k}$ & $0.22 \mathrm{~s} / 41 \mathrm{k}$ \\
\hline & $+m$ rods $)$ & & 4 & $2.61 \mathrm{~s}$ & $3.10 \mathrm{~s} / 187 \mathrm{k}$ & $2.69 \mathrm{~s} / 186 \mathrm{k}$ & $2.71 \mathrm{~s} / 186 \mathrm{k}$ & $1.42 \mathrm{~s} / 155 \mathrm{k}$ \\
\hline & & & 5 & $31.29 \mathrm{~s}$ & $41.47 \mathrm{~s} / 1042 \mathrm{k}$ & $37.03 \mathrm{~s} / 1039 \mathrm{k}$ & $36.89 \mathrm{~s} / 1039 \mathrm{k}$ & $18.67 \mathrm{~s} / 884 \mathrm{k}$ \\
\hline & & & 6 & $647.8 \mathrm{~s}$ & $951.5 \mathrm{~s} / 8228 \mathrm{k}$ & $866.9 \mathrm{~s} / 8191 \mathrm{k}$ & $839.3 \mathrm{~s} / 8191 \mathrm{k}$ & $461.8 \mathrm{~s} / 6941 \mathrm{k}$ \\
\hline & CSMA/CD & {$[1,1]$} & 2 & $\mathrm{O} / \mathrm{M}$ & $0.98 \mathrm{~s} / 42 \mathrm{k}^{*}$ & $1.47 \mathrm{~s} / 125 \mathrm{k}$ & $0.57 \mathrm{~s} / 34 \mathrm{k}$ & $0.56 \mathrm{~s} / 33 \mathrm{k}$ \\
\hline & (bus & & 3 & $\mathrm{O} / \mathrm{M}$ & $\mathrm{O} / \mathrm{M}$ & $5076 \mathrm{~s} / 2407 \mathrm{k}^{*}$ & $121.5 \mathrm{~s} / 807 \mathrm{k}$ & $0.66 \mathrm{~s} / 105 \mathrm{k}$ \\
\hline & $+m$ senders & & 4 & $\mathrm{O} / \mathrm{M}$ & $\mathrm{O} / \mathrm{M}$ & $\mathrm{O} / \mathrm{M}$ & $\mathrm{O} / \mathrm{M}$ & $2.47 \mathrm{~s} / 378 \mathrm{k}$ \\
\hline & & & 5 & $\mathrm{O} / \mathrm{M}$ & $\mathrm{O} / \mathrm{M}$ & $\mathrm{O} / \mathrm{M}$ & $\mathrm{O} / \mathrm{M}$ & $9.77 \mathrm{~s} / 1192 \mathrm{k}$ \\
\hline & & & 6 & $\mathrm{O} / \mathrm{M}$ & $\mathrm{O} / \mathrm{M}$ & $\mathrm{O} / \mathrm{M}$ & $\mathrm{O} / \mathrm{M}$ & $40.58 \mathrm{~s} / 3513 \mathrm{k}$ \\
\hline forward & Fischer's & same as & 2 & $0.34 \mathrm{~s}$ & $0.10 \mathrm{~s} / 20 \mathrm{k}$ & $0.10 \mathrm{~s} / 20 \mathrm{k}$ & $0.10 \mathrm{~s} / 19 \mathrm{k}$ & $0.08 \mathrm{~s} / 18 \mathrm{k}$ \\
\hline & mutual & above & 3 & $37.89 \mathrm{~s}$ & $\mathrm{O} / \mathrm{M}$ & $22.10 \mathrm{~s} / 561 \mathrm{k}$ & $19.18 \mathrm{~s} / 654 \mathrm{k}$ & $5.59 \mathrm{~s} / 538 \mathrm{k}$ \\
\hline & GRC & same as & 2 & $3.29 \mathrm{~s}$ & $2.29 \mathrm{~s} / 192 \mathrm{k}$ & $1.41 \mathrm{~s} / 95 \mathrm{k}$ & $1.43 \mathrm{~s} / 95 \mathrm{k}$ & $0.44 \mathrm{~s} / 84 \mathrm{k}$ \\
\hline & & above & 3 & $\mathrm{O} / \mathrm{M}$ & $\mathrm{O} / \mathrm{M}$ & $\mathrm{O} / \mathrm{M}$ & $\mathrm{O} / \mathrm{M}$ & $6.35 \mathrm{~s} / 418 \mathrm{k}$ \\
\hline & reactor & {$[1,1]$} & 2 & $\mathrm{O} / \mathrm{M}$ & $\mathrm{O} / \mathrm{M}$ & $\mathrm{O} / \mathrm{M}$ & $\mathrm{O} / \mathrm{M}$ & $\mathrm{O} / \mathrm{M}$ \\
\hline & CSMA/CD & {$[1,1]$} & 2 & $0.19 \mathrm{~s}$ & $0.19 \mathrm{~s} / 29 \mathrm{k}$ & $0.17 \mathrm{~s} / 29 \mathrm{k}$ & $0.17 \mathrm{~s} / 29 \mathrm{k}$ & $0.25 \mathrm{~s} / 33 \mathrm{k}$ \\
\hline & & & 3 & $2.63 \mathrm{~s}$ & $1.81 \mathrm{~s} / 102 \mathrm{k}$ & $1.64 \mathrm{~s} / 101 \mathrm{k}$ & $1.62 \mathrm{~s} / 101 \mathrm{k}$ & $2.61 \mathrm{~s} / 106 \mathrm{k}$ \\
\hline & & & & $68.75 \mathrm{~s}$ & $20.07 \mathrm{~s} / 370 \mathrm{k}$ & $17.49 \mathrm{~s} / 378 \mathrm{k}$ & $17.52 \mathrm{~s} / 378 \mathrm{k}$ & $27.03 \mathrm{~s} / 378 \mathrm{k}$ \\
\hline & & & & $\mathrm{O} / \mathrm{M}$ & $268.0 \mathrm{~s} / 1905 \mathrm{k}$ & $240.3 \mathrm{~s} / 1906 \mathrm{k}$ & $242.2 \mathrm{~s} / 1906 \mathrm{k}$ & $331.9 \mathrm{~s} / 1910 \mathrm{k}$ \\
\hline & & & & $\mathrm{O} / \mathrm{M}$ & $3889 \mathrm{~s} / 11725 \mathrm{k}$ & $3123 \mathrm{~s} / 11525 \mathrm{k}$ & $3155 \mathrm{~s} / 11525 \mathrm{k}$ & $4163 \mathrm{~s} / 11552 \mathrm{k}$ \\
\hline
\end{tabular}

s: seconds; k: kilobytes of memory in data-structure; O/M: Out of memory;

because they are already contained in the known parameter constraints $P$ and along each exploration path, the parameter constraints only get restricter.

PSPSC can help in pruning the space of exploration in big chunks. But in the worst case, PSPSC does not guarantee the exploration will terminate. In section 9, we shall report the performance of this technique. Especially, for one benchmark, the state-space exploration cannot converge without PSPSC.

\section{Implementation and Experiments}

We have implemented our ideas in our tool red which has been previously reported in [15-19] for the verification of timed automata. We have also carried out experiments and compared with HyTech 2.4.5 [13] and TReX 1.3 [1,3]. Details about the benchmarks can be found in our full version report. Experiments are conducted on a Pentium 4M 1.6GHz/256MB running LINUX.

Comparison with HyTech 2.4.5: We compare performance in both forward and backward reachability analyses. The performance data of HyTech 2.4.5 and red 5.0 with dictionary ordering (no PSPSC), coefficient ordering (no PSPSC), magnitude ordering (PSPSC), and coefficient ordering with PSPSC is reported in table 1. The experiment, although not extensive, does show signs that HRDtechnology (with or without PSPSC) can compete with the technology used 
Table 2. Performance comparison with TReX w.r.t. number of processes

\begin{tabular}{|c|c|c|c|c|c|c|c|}
\hline \multicolumn{2}{|c|}{ benchmarksconcurrency } & \multicolumn{3}{|c|}{ Forward } & \multicolumn{3}{|c|}{ Backward } \\
\hline & & TReX & red & 5.0 & TReX & red & 5.0 \\
\hline & & 1.3 & magnitude & oeff.+PSPSC & 1.3 & magnituder & oeff.+PSPSC \\
\hline Fiscl & 2 procs & $1.12 \mathrm{~s}$ & $0.07 \mathrm{~s} / 17 \mathrm{k}$ & $0.07 \mathrm{~s} / 15 \mathrm{k}$ & $8.96 \mathrm{~s}$ & $0.08 \mathrm{~s} / 13 \mathrm{k}$ & $0.04 \mathrm{~s} / 13 \mathrm{k}$ \\
\hline & 3 procs & $\mathrm{O} / \mathrm{M}$ & $1.86 \mathrm{~s} / 137 \mathrm{k}$ & $0.78 \mathrm{~s} / 79 \mathrm{k}$ & $\mathrm{N} / \mathrm{A}$ & $0.66 \mathrm{~s} / 43 \mathrm{k}$ & $0.49 \mathrm{~s} / 43 \mathrm{k}$ \\
\hline excl & 4 procs & $\mathrm{O} / \mathrm{M}$ & $197.9 \mathrm{~s} / 2714 \mathrm{k}$ & $16.92 \mathrm{~s} / 539 \mathrm{k}$ & $\mathrm{N} / \mathrm{A}$ & $5.81 \mathrm{~s} / 180 \mathrm{k}$ & $3.58 \mathrm{~s} / 158 \mathrm{k}$ \\
\hline & 5 procs & $\mathrm{O} / \mathrm{M}$ & $\mathrm{N} / \mathrm{A}$ & $752.7 \mathrm{~s} / 5254 \mathrm{k}$ & $\mathrm{N} / \mathrm{A}$ & $59.27 \mathrm{~s} / 945 \mathrm{k}$ & $24.71 \mathrm{~s} / 658 \mathrm{k}$ \\
\hline & 6 procs & $\mathrm{O} / \mathrm{M}$ & N/A & $\mathrm{N} / \mathrm{A}$ & $\mathrm{N} / \mathrm{A}$ & $567.1 \mathrm{~s} / 4341 \mathrm{k}$ & $170.3 \mathrm{~s} / 2798 \mathrm{k}$ \\
\hline reac & 2 rods & $\overline{\mathrm{O} / \mathrm{M}}$ & $\mathrm{O} / \mathrm{M}$ & $\mathrm{O} / \mathrm{M}$ & $\mathrm{N} / \mathrm{A}$ & $0.06 \mathrm{~s} / 19 \mathrm{k}$ & $0.05 \mathrm{~s} / 15 \mathrm{k}$ \\
\hline & 3 rods & $\mathrm{O} / \mathrm{M}$ & $\mathrm{O} / \mathrm{M}$ & $\mathrm{O} / \mathrm{M}$ & $\mathrm{N} / \mathrm{A}$ & $0.37 \mathrm{~s} / 52 \mathrm{k}$ & $0.22 \mathrm{~s} / 41 \mathrm{k}$ \\
\hline & 4 rods & $\mathrm{O} / \mathrm{M}$ & $\mathrm{O} / \mathrm{M}$ & $\mathrm{O} / \mathrm{M}$ & $\mathrm{N} / \mathrm{A}$ & $2.71 \mathrm{~s} / 186 \mathrm{k}$ & $1.42 \mathrm{~s} / 155 \mathrm{k}$ \\
\hline & 5 rods & $\mathrm{O} / \mathrm{M}$ & $\mathrm{O} / \mathrm{M}$ & $\mathrm{O} / \mathrm{M}$ & $\mathrm{N} / \mathrm{A}$ & $36.89 \mathrm{~s} / 1039 \mathrm{k}$ & $18.67 \mathrm{~s} / 884 \mathrm{k}$ \\
\hline & 6 rods & $\mathrm{O} / \mathrm{M}$ & $\mathrm{O} / \mathrm{M}$ & $\mathrm{O} / \mathrm{M}$ & $\mathrm{N} / \mathrm{A}$ & $839.3 \mathrm{~s} / 8191 \mathrm{k}$ & $461.8 \mathrm{~s} / 6941 \mathrm{k}$ \\
\hline
\end{tabular}

s: seconds; k: kilobytes of memory in data-structure;

O/M: Out of memory; N/A: not available;

in HyTech 2.4.5. For all the benchmarks, HRD-technology demonstrates better scalability w.r.t. concurrency complexity.

Finally, PSPSC cuts down the time and memory needed for parametric safety analysis. Especially, in forward analysis of the general railroad benchmark with three trains, without PSPSC, the state-space exploration fails to converge. This shows very good promise of this technique.

Comparison with TReX 1.3: Since TReX 1.3 only supports the verification of systems with clocks, parameters, and lossy channels, we choose the following two benchmarks. The first is Fischer's protocol with all clocks in the uniform rate of 1 . The second is the Nuclear Reactor Controller. The performance data is shown in table 2 for both forward and backward analysis.

Two additional options of red 5.0 were chosen: coefficient evaluation ordering with PSPSC and magnitude evaluation ordering without. We marked N/A not available) with higher concurrencies when we feel that too much time (like more than 1 hour) or too much memory (20MB) has been consumed in early fixpoint iterations. Although the data set is still small and incomplete, but we feel that the HRD-technology shows promise in the table.

\section{References}

1. A. Annichini, E. Asarin, A. Bouajjani. Symbolic Techniques for Parametric Reasoning about Counter and Clock Systems. CAV'2000, LNCS 1855, Springer-Verlag.

2. E. Asarin, M. Bozga, A. Kerbrat, O. Maler, A. Pnueli, A. Rasse. Data-Structures for the Verification of Timed Automatas. Proceedings, HART'97, LNCS 1201.

3. A. Annichini, A. Bouajjani, M. Sighireanu. TReX: A Tool for Reachability Analysis of Complex Systems. CAV'2001, LNCS, Springer-Verlag.

4. R. Alur, C.Courcoubetis, T.A. Henzinger, P.-H. Ho. Hybrid Automata: an Algorithmic Approach to the Specification and Verification of Hybrid Systems. Proceedings of HYBRID'93, LNCS 736, Springer-Verlag, 1993.

5. R. Alur, C. Courcoubetis, N. Halbwachs, T.A. Henzinger, P.-H. Ho, X. Nicollin, A. Olivero, J. Sifakis, S. Yovine. The Algorithmic Analysis of Hybrid Systems. Theoretical Computer Science 138(1995) 3-34, Elsevier Science B.V. 
6. R. Alur, T.A. Henzinger, P.-H. Ho. Automatic Symbolic Verification of Embedded Systems. in Proceedings of 1993 IEEE Real-Time System Symposium.

7. R. Alur, T.A. Henzinger, M.Y. Vardi. Parametric Real-Time Reasoning, in Proceedings, 25th ACM STOC, pp. 592-601.

8. F. Balarin. Approximate Reachability Analysis of Timed Automata. IEEE RTSS, 1996.

9. J.R. Burch, E.M. Clarke, K.L. McMillan, D.L.Dill, L.J. Hwang. Symbolic Model Checking: $10^{20}$ States and Beyond. IEEE LICS, 1990.

10. G. Behrmann, K.G. Larsen, J. Pearson, C. Weise, Wang Yi. Efficient Timed Reachability Analysis Using Clock Difference Diagrams. CAV'99, July, Trento, Italy, LNCS 1633, Springer-Verlag.

11. R.E. Bryant. Graph-based Algorithms for Boolean Function Manipulation, IEEE Trans. Comput., C-35(8), 1986.

12. D.L. Dill. Timing Assumptions and Verification of Finite-state Concurrent Systems. CAV'89, LNCS 407, Springer-Verlag.

13. T.A. Henzinger, P.-H. Ho, H. Wong-Toi. HyTech: The Next Generation. in Proceedings of 1995 IEEE Real-Time System Symposium.

14. J. Moller, J. Lichtenberg, H.R. Andersen, H. Hulgaard. Difference Decision Diagrams. In proceedings of Annual Conference of the European Association for Computer Science Logic (CSL), Sept. 1999, Madreid, Spain.

15. F. Wang. Efficient Data-Structure for Fully Symbolic Verification of Real-Time Software Systems. TACAS'2000; LNCS 1785, Springer-Verlag.

16. F. Wang. Region Encoding Diagram for Fully Symbolic Verification of Real-Time Systems. The 24th COMPSAC, Oct. 2000, Taipei, Taiwan, ROC, IEEE press.

17. F. Wang. RED: Model-checker for Timed Automata with Clock-Restriction Diagram. Workshop on Real-Time Tools, Aug. 2001, Technical Report 2001-014, ISSN 1404-3203, Dept. of Information Technology, Uppsala University.

18. F. Wang. Symbolic Verification of Complex Real-Time Systems with ClockRestriction Diagram. FORTE'2001, Kluwer; August 2001, Cheju Island, Korea.

19. F. Wang. Efficient Verification of Timed Automata with BDD-like Data-Structures, to appear in special issue of STTT (Software Tools for Technology Transfer, Springer-Verlag) for VMCAI'2003, LNCS 2575, Springer-Verlag.

20. F. Wang. Model-Checking Distributed Real-Time Systems with States, Events, and Multiple Fairness Assumptions. 10th AMAST, LNCS, Springer-Verlag.

21. F. Wang, G.-D. Hwang, F. Yu. TCTL Inevitability Analysis of Dense-Time Systems. 8th CIAA, LNCS 2759, Springer-Verlag.

22. F. Wang, A. Mok, E.A. Emerson. Symbolic Model-Checking for Distributed RealTime Systems. 1st FME, April 1993, Denmark; LNCS 670, Springer-Verlag.

23. H. Wong-Toi. Symbolic Approximations for Verifying Real-Time Systems. Ph.D. thesis, Stanford University, 1995. 